\title{
Carnets
}

Revue électronique d'études françaises de l'APEF

Deuxième série - 17 | 2019

Lîlle : prisme de la connaissance ou reconnaissance du monde

\section{La réalité dystopique de l'idéologie nazie : entre imagination et inintelligibilité}

\section{Atinati Mamatsashvili}

\section{OpenEdition}

Journals

Édition électronique

URL : http://journals.openedition.org/carnets/10435

DOI : $10.4000 /$ carnets. 10435

ISSN : 1646-7698

Éditeur

APEF

Référence électronique

Atinati Mamatsashvili, «La réalité dystopique de l'idéologie nazie : entre imagination et

inintelligibilité », Carnets [En ligne], Deuxième série - 17 | 2019, mis en ligne le 30 novembre 2019,

consulté le 07 janvier 2020. URL : http://journals.openedition.org/carnets/10435 ; DOI : 10.4000/ carnets. 10435

Ce document a été généré automatiquement le 7 janvier 2020.

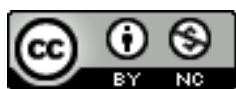

Carnets est mis à disposition selon les termes de la licence Creative Commons - Atribution - Pas d'utilisation commerciale 4.0 International. 


\title{
La réalité dystopique de l'idéologie nazie : entre imagination et inintelligibilité
}

\author{
Atinati Mamatsashvili
}

\section{NOTE DE L'AUTEUR}

Ce travail est réalisé dans le cadre de la Bourse de la Fondation pour la Mémoire de la Shoah.

1 La faculté d'imagination apparaît cruciale dans la non-domestication de la pensée par des régimes totalitaires. Les anti-utopies du vingtième siècle ont bien saisi l'importance de la faculté imaginative qui se présente souvent comme une menace de premier ordre à l'État total, ce dernier s'efforçant de la supprimer ${ }^{1}$ (Zamiatine, 2010).

2 Nous envisageons d'interroger le regard porté par les écrivains français sur l'Allemagne nazie, souvent par le biais du voyage qu'ils ont réalisé eux-mêmes entre 1933 et 1939. La question à se poser est la suivante - étant devenus témoins directs, ont-ils appréhendé le danger et la menace de l'état dictatorial, des lois raciales, des persécutions des Juifs ? Sous quelle forme littéraire ont-ils dénoncé ou annoncé les périls existants ou à venir ? Quelle était l'image, réelle ou imaginée, de cette Allemagne à l'emprise des défilés, des saluts nazis, des drapeaux à la croix gammées hissés partout dans les rues? Était-il possible d'imaginer ou d'appréhender la réalité dystopique qui se présentait à eux ? Quelle était la marge entre la réalité visuelle déployée et l'inconcevabilité de cette réalité inimaginable?

En 1940 Erika Mann publie aux États-Unis un recueil de nouvelles intitulé Quand les lumières s'éteignent. Il s'ouvre sur l'arrivée d'un étranger dans une petite ville d'Allemagne à peine avant la déclaration de la Deuxième Guerre mondiale. Le récit se focalise sur la visite la ville allemande par un américain qui est une sorte de "voyage d'exploration » et qui aboutit au constat suivant : «Je pourrais rester ici des jours, des 
semaines et des mois entiers sans connaître la ville» (Mann, $2011: 59)$. Confronté à la réalité tangible (car le voyageur voit tout de ses propres yeux, sans intermédiaire), le seul repère qu'il a pour interpréter ce qu'il voit, ce sont des informations obtenues dans les journaux lorsqu'il se trouvait en dehors du lieu visité. Ces informations sont entièrement négatives ("atrocités envers les Juifs", Mann, 2011: 53 ; «Terreur de la dictature ", Mann, $2011: 43$ ). En réalité, ces repères lui servent à renier l'image funeste de l'Allemagne et de la remplacer par celle qui est au contraire "envoutante » et " charmante» (Ibid.), et surtout bénéfique pour le peuple allemand:

Ce qui figure dans les journaux est simple et sans ambiguïté. Selon la volonté du Führer, les Juifs et les communistes doivent être éliminés. Il est vrai que ce n'est pas très joli, mais si c'est pour le bien du peuple, alors c'est le prix à payer. En outre la nation est unie sous le signe de l'amour de la patrie et de sa réhabilitation » (Mann, 2011: 58).

4 Le récit nous intéresse dans la mesure où il dévoile, par le biais du personnage de l'étranger placé dans un espace non familier et dystopique ${ }^{2}$, une inaptitude absolue de la faculté de l'imagination au sens où l'entend Hannah Arendt, lorsqu'elle parle d'«absence de pensée » et de «manque d'imagination » qui se posent à l'amont de la faille de l'argumentation chez Eichmann (Arendt, 2002). Nous l'entendons aussi au sens que lui est conféré par Victor Klemperer lorsqu'il s'en rapporte à la faculté imaginative d'une jeune fille «au nom lituanien » qui n'arrive pas à généraliser le problème - en protestant contre l'infériorité de sa "race » par rapport à la race aryenne, et qui déclare simultanément que les Juifs « c'est autre chose », que cette fois-ci, le Führer a raison ${ }^{3}$.

5 Ce qui attire l'attention dans le récit d'Érika Mann, ce n'est pas tant le sens de conformisme ou d'adaptation totale à l'atmosphère étrangère de la part du voyageur, comme par exemple prononcer « Heil Hitler » de son propre gré et de faire le salut nazi (Mann, 2011: 54); ce ne sont pas non plus ses paroles horribles lorsqu'il approuve le programme d'élimination des Juifs dans le but du bien de la nation, mais ce qui frappe avant tout c'est l'impossibilité d'intelligibilité et de mise en relation des faits observés. Car, en réalité, l'étranger, au cours de sa courte visite, s'est heurté à tous les degrés de l'idéologie nazie, personne ne lui a caché les camps de concentrations, les affiches et les slogans, l'armement, le degré d'endoctrinement, la peur des dénonciations auquel le peuple est sujet. Néanmoins, il prétend ne pas pouvoir « connaître » le pays, même s'il y restait des mois. Ce qui se dévoile à travers la fabula, c'est ce refoulement de ne pas vouloir connaître, car imaginer l'ensemble de ces faits, implique autant une part de responsabilisation. Imaginer la réalité dans sa véracité de faits, est aussi endosser la responsabilité de tout ce que cela implique.

\section{Voyager " en terre brune " : quels repères pour l'étranger de passage?}

6 Dans son étude consacrée aux voyages dans l'Allemagne nazie entre 1933 et 1939, Frédéric Sallée souligne dans quelle mesure «l'antisémitisme nazi est minimisé par le voyageur car intégré dans un ensemble mondial de persécutions » (Sallée, 2017 : 338). L'antisémitisme, même étant devenu « la vision du monde » nazie, n'est pas en soi une nouveauté pour des sociétés européennes des années trente. Pour cette raison, le voyageur «n'a pour outil de mesure que l'image que lui renvoie le traitement de 
l'antisémitisme dans son propre pays » (Sallée, 2017 : 330). Il est certain qu'en France l'antisémitisme a été particulièrement présent dans le paysage politique des années trente. En outre, la question de l'antisémitisme ne se perçoit pas comme quelque chose de singulier, mais se dilue dans le paysage global des répressions perpétrées par l'État nazi ${ }^{4}$. C'est la raison pour laquelle rares sont les témoignages (dont récits de voyage, romans etc.) qui rendent compte, dès les années trente, de la réalité où se décèle l'image réelle à travers l'image délibérément fausse dont veut user le Reich devant le voyageur étranger. Par exemple, durant les jeux Olympiques, l'Allemagne prend toutes sortes de mesures concernant son traitement "adéquat de la question juive ", où le «traitement adéquat» signifie de convaincre de l'«hospitalité allemande» les "étrangers qui n'ont malheureusement pas encore tous une conception juste de la nouvelle Allemagne » (circulaire de 1936, cité in Sallée, $2017: 341$ ) ; ainsi, seront prises des directives spéciales, comme par exemple, seront déplacés les placards ou affiches concernant les Juifs afin de les dissimuler. Mais que voit le voyageur étranger lorsqu'il voyage en Allemagne et lorsque cette dissimulation n'a pas eu lieu? Que perçoit son imagination à travers les étendards, les défilés, les interdictions, des persécutions, les émissions radiophoniques? Peut-il imaginer, ne serait-ce un instant, cette réalité dystopique dans sa réalité réelle?

7 Lorsque Pierre Frederix, journaliste et écrivain qui a effectué, entre 1933 et 1936, plusieurs séjours de longue durée en Allemagne pour Le Petit Parisien, s'interroge dans son ultime article sur la réalité allemande, il note: «Mon impression dominante sur l'Allemagne actuelle, après sept semaines de séjour? Celle d'une confusion mentale complète » (Frederix, 1934: 1). Cette confusion mentale ou autrement dit, cette incompréhension, provient d'une totale « contradiction » (Frederix, 1934:3) dont tout fait preuve dans ce pays "naziste» (Ibid.) : «Ainsi les paroles ne s'accordent pas plus entre elles qu'elles ne s'accordent avec les écrits ni avec les réalités » (Ibid.), tout comme " aucun mot n'y a de sens fixe » (Ibid.). Pour lui, le peuple allemand « se plaît » "dans l'abstraction ou dans la mythologie » (Ibid.), dans ce qui ne peut être qualifié, déjà en 1934, que de "pure utopie » (Ibid.). "L'irrationalité de la pensée nazie, comme le remarque Sallée, déforme les contours de la pensée classique rationnelle $»^{5}$ (Sallée, 2017 : 58). Partagée entre «confusion mentale » ou «mystère » (Beucler, 1933: 1), le phénomène nazi, avec son idéologie antisémite et la morale des persécutions qui se justifient par la nécessité vitale nationale, se traduit donc souvent par l'impossibilité de compréhension chez l'écrivain ou le journaliste qui s'y rend sur place. Même s'il ne s'agit pas souvent de lucidité de regard que ces derniers lui portent, nous allons nous centrer sur cette perspective qui éclaire sur le danger et la nouveauté du phénomène.

Ce ne furent point les critiques habituelles contre les Israélites que j'entendis alors. Ce n'était même point une attaque à laquelle j'assistais. C'était le développement d'une théorie grandiose, d'une abracadabrante histoire universelle naziste, commençant au VIe siècle avant J.C. par le massacre de 75000 personnes sur l'ordre d'un ministère des Finances juifs du shah de Perse et finissant par la ligne téléphonique privée qui permettait à Rathenau de donner à Guillaume II les ordres des Sages de Sion » (Frederix, 1933, p. 5).

8 En 1926 déjà, l'écrivain et journaliste Henri Béraud soulignait le caractère particulier de l'antisémitisme en Allemagne pré-hitlérienne qui se décelait dans la visée exterminatoire des adeptes de Hitler :

Nous n'avons aucune idée, en France, de ce que peut être l'antisémitisme des réactionnaires allemands. Ce n'est ni une opinion, ni un sentiment ni même une réaction physique. C'est une passion, une véritable obsession d'intoxiqués et qui 
peut aller jusqu'au crime (...). Les racistes rêvent pire encore. Ils sont les Aryens contre les Sémites, et ils se voudraient des âmes d'exterminateurs ${ }^{6}$ (Béraud, 1926 :

1).

9 Il est notoire dans quelle mesure l'attention était attirée sur le caractère de l'idéologie nazie reposant spécifiquement sur la doctrine antisémite. C'est avant tout au niveau de la forme que nous allons nous interroger quant à l'explicitation du phénomène par le regard étranger. Il s'agit souvent d'une tentative de définition qui passe par la négativité. C'est-à-dire, avant de formuler une opinion, afin que le lecteurinterlocuteur puisse imaginer le phénomène nouveau qui l'est aussi pour son locuteur (qui n'est d'ailleurs pas sûre d'avoir compris lui-même la chose), ce dernier procède par détailler ce que cette nouveauté ne représente pas. L'antisémitisme des hitlériens, écrit Béraud, n'est ni une opinion, ni un sentiment, ni une réaction physique. Il faut donc faire imaginer une chose inexistante quelque part, ce dont ils n'auraient «aucune idée » en France (selon le propos de Béraud). Ce que Frederix entend en Allemagne, ce ne sont pas des critiques habituelles contre les Juifs, ni une attaque à leur encontre, c'est quelque chose de différent qui s'ancre dans une idéologisation de la pensée humaine ${ }^{7}$.

Le témoin, afin de faire figurer ce qui n'est pas habituel, il faut qu'il puisse premièrement le décrire. Le locuteur, à l'instar de ce dernier, il faut qu'il puisse imaginer ce qui est décrit, si ce dernier ne repose sur aucun repère existant au préalable. C'est aussi sans doute l'une des causes pour lesquelles le voyageur-écrivain (ainsi que le locuteur) n'arrive pas à décrypter le danger du nouvel antisémitisme (car, il le considère par rapport au système de fonctionnement qu'il connait, qui lui est familier, et comme l'antisémitisme est très présent en France des années trente, ce fait l'empêche notamment d'appréhender le mode de fonctionnement nouveau). Dès lors, le familier s'avère être paradoxalement un facteur d'empêchement à l'intelligibilité. Par ailleurs, une autre cause pour le voyageur-écrivain de ne pas être en mesure d'imaginer l'ampleur du phénomène réside dans le fait que ce dernier est sans précédent. Dans les deux cas, le familier fonctionne comme obstacle à l'imagination de la réalité dystopique ${ }^{8}$.

\section{Ne pas comprendre, mais imaginer : la construction du refus à travers l'inintelligibilité}

11 Afin de mieux cerner la question de l'intelligibilité, liée à la faculté perceptive et descriptive simultanément, nous allons nous focaliser sur le texte fictionnel d'André Chamson, L'année des vaincus, écrit en 1933-1934, en quelques mois seulement. Le livre a été surtout inspiré par les événements du 6 février 1934. Pourtant, son auteur, qui ne l'a pas publié tout de suite en pensant que la paix était toujours possible, s'y décide un peu plus tard, poussé par «l'agressivité hitlérienne " (Cellier-Gelly in Chamson 2005 : V). Nous allons nous focaliser brièvement sur l'épisode qui met en scène la rencontre entre le personnage-mineur, Carrière, ayant fait un court voyage en l'Allemagne de Hitler et ses camarades qui l'interrogent, à son retour, sur ses impressions (Chamson, 2005 : 94-104). Il s'agit d'une mise en perspective de l'imaginaire (ce qui est imaginé par les villageois sur l'Allemagne en fonction de ce qu'ils entendent autour d'eux, ce qu'ils lisent dans les journaux etc.) et des faits réels (ce que le voyageur a vu de ses propres yeux en se rendant sur place). Mais c'est notamment ce lieu de rencontre entre 
l'imaginaire et le réel qui s'avère se rendre problématique. La difficulté se traduit sur plusieurs niveaux : (1) l'inintelligibilité de l'événement (du nazisme) par le protagoniste (Carrière) qui effectue le voyage, mais qui pourtant perçoit son anormalité ; (2) deuxièmement, cette inintelligibilité conduit à l'impossibilité de nommer l'événement (c. a. d. impossible d'expliquer ce qui précisément n'est pas normal) ; (3) troisièmement, si la nomination devient impossible, c'est parce que l'apparence, à son état descriptif, ne rend pas l'essence de la chose que représente le nazisme. (4) De ce fait (et c'est notre quatrième point), le protagoniste se plonge dans le mutisme et esquive tout contact avec les amis afin de ne pas avoir à parler de ce qu'il a vu. Comme sa fuite ne peut pas être interminable, ils arrivent en fin de compte à le forcer à raconter. Cette scène renvoie à l'interrogatoire: eux, installés en face de lui, autour de la table, avec une lumière au-dessus qui éclaire Carrière. Pour répondre à leurs regards pleins d'attente dirigés vers lui et à la question impatiente qui les tourmente - "Alors? (Chamson, 2005 : 95), la première parole lâchée par Carrière est la suivante : "- Alors? Je suis contre. Voilà » (Chamson, 2005 : 95). À ce propos, «Les six mains, ouvertes vers lui, soulevées par l'attente, retombèrent sur le tapis à dessins jaunes. Elles se fermaient lentement, dans une crispation des doigts et de la paume » (Ibid.). Le premier contact entre l'imaginaire et le réel consiste dans la négation totale, le rejet total de l'événement nazi, même dans son inintelligibilitée . Et c'est ici que réside la différence de perception entre Carrière et ceux qui ne comprennent pas, mais toutefois soit $\mathrm{y}$ décèlent la normalité soit plongent dans un acquiescement entier, alors que Carrière rejette le phénomène. Mais ce rejet ne signifie pas simultanément la compréhension et l'aptitude de description.

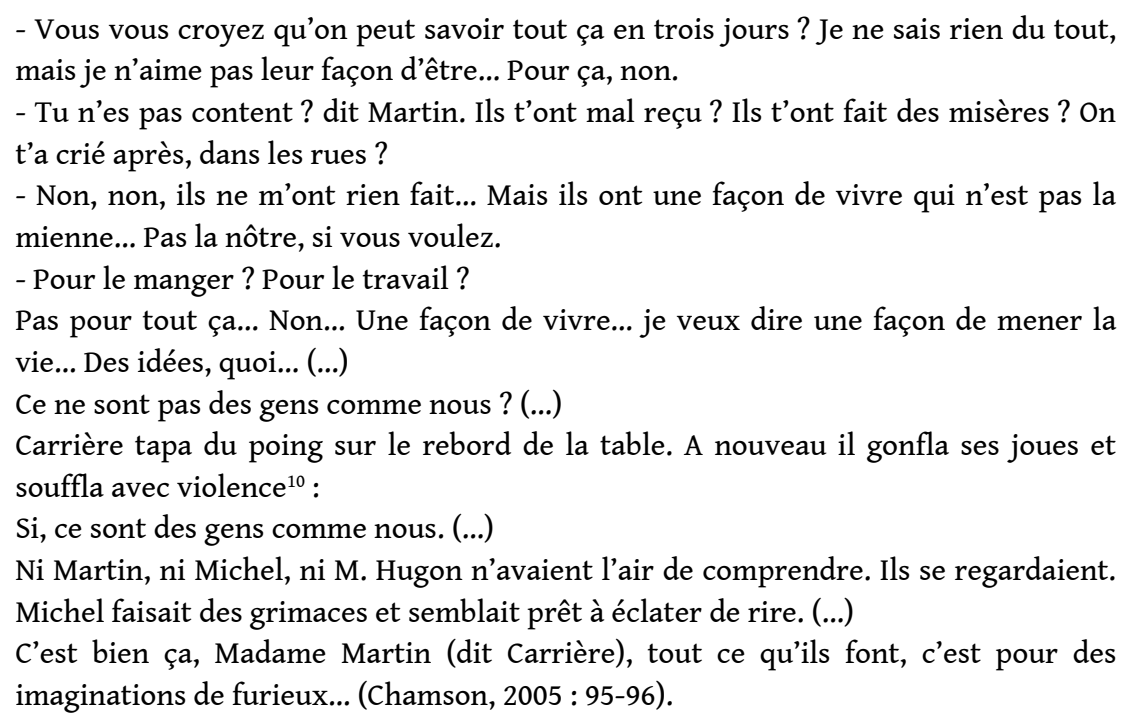

L'explication procède ici par la négation. Même si l'explication échoue pour dire ce que ça représente, Carrière sait néanmoins ce que cette chose ne représente pas. Cette certitude est suivie d'une autre : il refuse, il n'est pas d'accord, il n'admet pas cette réalité inintelligible. Une fois de plus, le concept de l'imagination intervient («tout ce qu'ils font, c'est pour des imaginations de furieux»), cette fois dans son rapport à l'irrationnel, au mythologique (le mot n'est pas nommé). Le mode de vie des Allemands bascule donc vers "des imaginations de furieux» qui se traduit dans chaque geste banal, comme par exemple, boire un verre s'égale à boire un verre "pour la grandeur de l'Allemagne » (Chamson, 2005 : 96). Pourtant, il ne s'agit pas de la folie mentale, ils conservent le sens de jugement du bon et du mauvais (Chamson, 2005 : 97). La tentative 
d'explication se heurte à une impossibilité d'imaginer par les autres les faits décrits par le protagoniste. En fin de compte l'inaptitude d'imaginer se transforme en rire (comme personne n'arrive à comprendre les propos de Carrière, ils commencent à rire) et qualifie la parole du témoin - de folie. L'incapacité d'expliquer se situe dans cet espace langagier où la norme, sans toutefois «modifier les codifications" (Jouanjan, 2017 : 288) qui la définissent, se façonne par un renversement radical de concepts et de notions.

米 nazi. Comme l'Histoire. Comme l'Économie. » (Dorgelès, 1937 : 5). Le fonctionnement de ce monde nazi repose sur un mode d'être dans laquelle «il est immoral qu'un juif puisse disposer de la pensée aryenne" (cité par Dorgelès, $1937: 1$ ). Ce qui trouble le plus cet écrivain dans le traitement infligé aux Juifs, c'est «moins l'iniquité des mesures qu'on leur applique que leur stupidité. Qu'un pays de haute civilisation comme l'Allemagne puisse en arriver là, cela dépasse l'entendement " (Dorgelès, 1937: 1). L'inintelligibilité ou le choc se situe donc moins au niveau fonctionnel qu'au niveau idéologique ; c'est-à-dire, ce qui est inintelligible ce n'est pas tellement l'iniquité des mesures en soi, mais la conception de ces mesures, l'idée fondatrice qui fait qu'on les invente et les applique. Dorgelès s'interroge par conséquent non pas tellement sur le fonctionnement de l'État nazi par le biais des personnes physiques qui le font maintenir, mais davantage sur la genèse de ces mesures. «Interdit à un juif de posséder des terres. Interdit à un juif de plaider pour des Aryens. Interdit à un juif d'être fonctionnaire. Interdit à un juif d'arracher des dents ou de faire du cinéma, à moins qu'il ne soit décoré à titre de guerre ou que son père ait été tué au front. (...) Ils sont rayés des cadres de la nation » (Dorgelès, $1937: 1$ ). Ainsi, devenus "parias », ils sont dépourvus de tous droits. Le nouveau Code intervient uniquement pour leur interdire ou les interdire tout court. "On ne les traite pas tout à fait comme des bêtes, soyons justes. Mais comme des hommes encore moins » (Ibid.). Plus tard, lorsque ces mesures d'exclusion vont frapper les Juifs dans son propre pays, sous l'Occupation, Dorgelès dépeint, pour s'y opposer, dans son récit intitulé la Carte d'identité, les décrets discriminatoires de Vichy, le port de «l'étoile jaune, l'internement, le cachot» (Dorgelès, 2013 : 926). Il pointe sur la vulnérabilité de ceux qui deviennent la cible par excellence du nouveau régime : «[...] ils ne parvenaient pas à pincer sur le fait ceux qui traçaient de grands $\mathrm{V}$ sur les murs. Ils soupçonnaient bien le curé lorrain mais un prêtre, malgré tout, cela s'arrête moins facilement qu'un Juif » (Dorgelès, 2013 : 928). Publié juste après la Libération, en 1945, la Carte d'identité retrace l'Histoire et désigne la responsabilité de Vichy, mais évoque aussi la bestialité des soldats allemands dans la traque et la persécution de ceux considérés à présent comme marginaux - «des terroristes, des Juifs, des étrangers » (Dorgelès, 2013 : 937).

Dans un autre article paru en janvier 1937, Dorgelès raconte dans quelle mesure la peur de dénonciations guette les gens non seulement dehors, mais aussi chez eux. Ici, il introduit de nouveaux termes qui sont sans doute absolument inconnus au public français. Il s'agit de «block-wart» qui est, selon Dorgelès, "encore une curieuse invention que celle de ce mouchard officiel. Le block-wart est chargé de surveiller un immeuble ou un groupe de maisons au nom du Parti» (Dorgelès, 1937a: 3). Cette 
surveillance permanente entretient une terreur permanente. André Beucler, lors de ses reportages sur l'Allemagne en 1933, souligne déjà à cette époque la terreur qui saisit toute la famille lorsqu'elle entend quelqu'un monter «les escaliers à pas lourds » et lorsqu'ils comprennent un peu plus tard que les pas ont dépassé leur porte: le père, "blême ", « ne me tendit pas la main. Le cousin israélite avait disparu ; les jeunes filles poussèrent un soupir de soulagement. On était ravi de voir que je cherchais à me lever » (Beucler, $1933: 5)^{11}$. Roland Dorgelès, ayant introduit un nouveau terme de " block-wart", l'accompagne aussitôt de l'autre, "Schutzhaft", encore moins compréhensible qui signifie dans le langage national-socialiste une détention de protection ou comme l'écrit Dorgelès : "pour vous protéger ». Enfin, il rapporte sa conversation avec ses hôtes qui lui expliquent qu'à côté de leur loge se trouve une liste des locataires indiquant «leur nationalité, leur profession et, c'est plus fort, leur religion. Comprenez-vous ? Notre hôtesse, à ces mots, regarda le ciel d'un œil mouillé : Mon Dieu, heureusement encore que nous ne sommes pas juifs $»^{12}$ (Dorgelès, 1937a: 3 ). C'est sur ces mots que Dorgelès termine l'article, sans y joindre aucun commentaire.

\section{Ville imaginée, ville réelle}

Philisterburg - tel est le titre que Jacques Decour donne à son récit qu'il ramène de son voyage en Allemagne effectué entre 1930-1931. L'intitulé est le nom de la ville dans laquelle se déploient les événements. Le nom imaginé pour une ville réelle, Magdebourg. C'est à Magdebourg que l'auteur est nommé comme professeur d'échange dans un lycée, ou plus précisément, comme assistant lecteur de français. L'action se déroule dans Philisterburg, la capitale des Philistins, quelque deux ans avant l'avènement de Hitler au pouvoir. Le livre, paru en 1932, précédé un peu avant par une publication d'extraits dans Annales, déclenche toute une série de polémiques, « heurtant avant l'heure la future bonne conscience collaborationniste» (Favre, 2002: 61) et provoquant la «malveillance des autorités 'sorbonnardes'« (Favre, 2002:62). Peut-on qualifier le texte de récit? ou plutôt de récit de voyage? ou peut-être de journal ? Decour s'attarde lui-même sur le genre : il s'agirait selon lui de "petits faits vrais » (Decour, $2003:$ p. 36) qui ont pour but non pas « le désir de persuader » (Decour, 2003 : 37), mais de rapporter les faits et de formuler de temps à autre une opinion. Il n'a pas «l'intention de faire une œuvre d'art» (Decour, 2003: 39). Nous ne pouvons pas parler de journal non plus - les dates sont très rarement formulées. Selon Paulhan, c'était « le récit le plus simple qui soit » (cité in Favre, $2002: 58$ ).

16 Ce qui nous intéresse dans ce texte extraordinairement lucide et de ce fait si scandaleux à l'époque et qui a failli influer sur la carrière de son jeune auteur (reçu parmi les premiers à l'agrégation, Decour est reconnu « agrégé des Lycées » trois mois plus tard), c'est d'une part le regard porté sur l'Allemagne, regard étranger et intérieur à la fois (étant sur place, connaissant la langue); d'autre part, c'est la mise en relief des repères qui permettent à son auteur de ne pas se prêter à un jugement préconçu (formulé dans les manuels d'histoire, les journaux français, dans l'opinion publique), parmi lesquels se trouvent ses propres convictions, comme par exemple, son dénie du nationalisme: "Je suis vacciné contre la peste du nationalisme» (Decour, 2003 : 37). Dès lors, c'est par rapport à ces repères que l'observation de la ville est faite. Pourtant, un passage interpelle le lecteur dans la mesure où il s'agit de l'effacement de repères qui contribuent au quasi-basculement dans l'attrait du spectacle d'un défilé nazi : 
(...) À ma fenêtre, je n'étais déjà plus simple spectateur. J'étais pénétré par le rythme uni du chant et du pas. Je n'avais plus très envie de sourire; j'étais peu à peu entrainé par le sentiment collectif qui les animait ; pour un peu, j'aurais chanté avec eux. Je ne trouvais plus très ridicule la petite bonne qui, du bord du trottoir, tenait depuis cinq minutes son bras droit levé pour les saluer; au passage ils répondaient, sans un mot, par le salut fasciste $»^{13}$ (Decour, 2003 : 68-69).

Ce bref passage prolonge sans doute la dialectique des défilés dans L'année des vaincus. La répulsion du protagoniste chamsonien à l'égard de ce qu'il va voir et dont il va être témoin, est ici pris à rebours : le témoin-observateur se trouve d'un seul trait démuni de tous ses repères. Le pas rythmé et uni du « régiment civil » qui cantonne un chant « mi-religieux », " mi-guerrier» (Decour, 2003: 68), l'entraine dans l'élan collectif. Victor Klemperer aurait parlé d'"une seule valse» et d'un "corps unique" (Klemperer, 1996: 43) dont le spectacle est apte à faire basculer le spectateur dans "l'extase» (Klemperer, 1996: 44). La phrase chez Decour, "pour un peu, j'aurais chanté avec eux ", ne pouvait bien évidemment avoir le sens identique pour le lecteur français de l'époque (car le livre est destiné au public français), à celui qu'elle acquiert, aujourd'hui, rétrospectivement. C'est aussi en ce sens que nous intéressent les deux textes, celui de Chamson et celui de Decour, étant quasi-contemporains l'un à l'autre. Decour décrit l'événement observé de sa fenêtre et rapporte ensuite sa propre réaction qui faillit ici passer d'une observation à l'implication sans que le passage soit explicité pour un lecteur extérieur. Il décrit une sorte d'horreur avec une lucidité précise. Tandis que chez Chamson ce qui est justement négligé, c'est la description, car c'est cette dernière qui échoue : l'impossibilité de décrire ce qui est observé d'une part et ce qui est ressenti (répulsion inexpliquée) d'autre part; cette incapacité est confrontée en fin de compte à l'impossibilité de compréhension par l'autre à qui l'événement est raconté. Donc ce qui manque ici, c'est l'événement imaginé dans les deux cas. Par conséquent, le refus est triplé : refus d'imaginer, refus de comprendre, refus de décrire. Chez Decour, par le biais de cette limite entre observation-participation, l'imagination est dépassée : l'observateur-témoin arrive à s'y projeter et désormais il peut imaginer et par conséquent - décrire. Chamson, au contraire, omet la parenthèse et montre l'inintelligibilité (et sa négation), alors que Decour s'attarde sur la parenthèse qui relie le descriptif au résultat (refus d'adhérer, à l'instar du protagoniste chamsonien) ${ }^{14}$.

Dans cette ville « imaginaire ", l'imagination n'a pas de place. À la question, ce que l'un des élèves reçoit à Noël comme cadeau parental, la réponse est : «- Des livres. - De la littérature? - Ah! Non! Je ne lis que de l'histoire et de la politique» (Decour, 2003 : 130). En effet, la littérature paraît déjà être bannie de la vision du monde des jeunes de dix-sept ans. Dans "Goethe et la jeunesse allemande» Decour note combien ils préfèrent «à la lecture d'une poésie de Goethe celle d'un journal bien informé et violemment tendancieux. La littérature ne les intéresse plus $»^{15}$ (Decour, $\left.2003: 167\right)$. En effet, la littérature, cette matière à réflexion qui appelle à l'imagination, ne s'accorde pas avec la weltanschauung qui néglige l'individu au profit du collectif et aspire à la "'race pure' et unie» (Decour, 2003 : 166). Dans ces conditions, le décalage entre la question et la réponse acquiert un sens encore plus révélateur : «- Que pensez-vous de Heine ? leur ai-je demandé. - Heine n'est pas Allemand. - Pourquoi ? - Il est Juif » (Decour, 2003 : 123) ; un peu plus loin : «Beethoven, quand est-il né ? » a pour réponse logique - «Beethoven, ce n'est pas un Allemand» (Decour, $2003: 127)$. En effet, comme paraphrase Decour, «Pas de Juifs ni de poètes équivoques!» (Decour, 2003: 166). Ce décalage dénué de toute logique entre la question et la réponse, révèle dans quelle 
mesure l'absence d'imagination y prend la fonction principale, au sens souligné plus haut, et qui se focalise comme signe annonciateur des événements néfastes quelques années plus tard.

Dans le raisonnement de Decour, cette conception d'imagination est opposée à celle qui renvoie à la connaissance : «C'est une de ces maisons presque toutes semblables de la rue Bismarck, avec des cactées poussiéreuses derrière les fenêtres toujours fermées. [...] J'essayais d'imaginer les passions, les drames des habitants énigmatiques. Je rêvais de connaître l'essence de leurs pensées $»^{16}$. (Decour, 2003 : 59) La maîtrise de la langue, de la littérature, n'efface pas l'étrangeté, la différence ou «l'énigme » qu'habite le lieu. Mais ceci est doublé par le désir de connaissance qui passe par l'imagination : imaginer derrière les fenêtres closes le secret des habitants, leur vie quotidienne ou encore imaginer ce qu'ils pensent et « comment ils pensent » (Decour, $2003: 48$ ).

\title{
Conclusion
}

\author{
«Il me parlait avec authenticité de Goethe et de \\ Heine. Il avait un teint olivâtre, des yeux luisants, \\ des boucles brunes : c'était un Juif. \\ - Savez-vous, disait-il, que je suis persécuté? \\ - À cause de votre 'race', ou parce que vous lisez \\ Goethe?» \\ (« Goethe et la jeunesse allemande », Decour, \\ $2003: 166)$
}

Dans le but de démontrer le lien entre l'art et la morale et en s'appuyant sur l'exemple des Mangeurs des pommes de terre de Van Gogh, Mattew Kieran met l'accent sur l'imagination comme élément central dans la compréhension du tableau. La manière dont le spectateur peut "imaginer " l'attention que les paysans démontrent les uns envers les autres, étant eux-mêmes dans le besoin, peut influer sur sa propre relation avec les autres (Kieran, 1996: 346). C'est ainsi que l'art favorise "la compréhension imaginative " (Kieran, 1996 : 346) précisément parce que la manière dont quelque chose est représentée "façonne en partie ce qui doit être imaginé " et par conséquent, appréhendé : « Art cultivates our imaginative understanding in a distinctive way, a way in which our ordinary imaginings cannot»(Kieran, 1996:346). Ce raisonnement développé par Kieran n'est pas sans lien avec celui qui se dessine à travers le récit de Decour et d'une certaine façon, celui de Chamson. L'art et la littérature, sans forcément mettre l'accent sur la charge morale, appellent à l'imagination de l'autre, autrement dit, à la « compréhension imaginative » qui est modelée au contact de l'œuvre d'art et qui ne pourrait se réaliser à travers aucune autre expérience imaginative ordinaire. Par conséquent, le déni de littérature dans l'Allemagne pré-hitlérienne, fonctionne dans Philisterburg comme un avant-texte de l'abolition de l'imagination, de cette forme particulière de compréhension qui aboutit à l'incendie d'œuvres d'art sur une place publique. La phrase qui met sur un même plan la persécution pour cause raciale et pour cause littéraire (" À cause de votre 'race', ou parce que vous lisez Goethe?»), n'a en fin de compte rien d'ironique, mais dévoile cette vérité imaginée, qui, comme l'aurait exprimé Magritte, «n'évoque rien d'imaginaire, [elle] évoque la réalité du monde que l'expérience et la raison considèrent confusément » (Magritte in Foucault, 1973 : 89). 
21 Reprise dans un autre sens, le terme de "confusion " revient sous la plume des journalistes (Frederix) qui essaient de façonner en termes intelligibles l'image qui se déploie sous leurs yeux. Cette « confusion mentale » survient en effet du choc entre la réalité et l'impossibilité d'imaginer cette même réalité dans sa factualité qui devient une réalité dystopique. Chamson, quoique inspiré en premier lieu du contexte français (les événements du 6 février 1934), construit son récit autour de l'inintelligibilité de l'image déplacée hors frontière (le protagoniste effectue le voyage en Allemagne) et ensuite réintégrée au contexte familier (le protagoniste revient en France et dépeint ce qu'il a pu observer). Ici, le double échec de compréhension (par le protagoniste et par ses interlocuteurs) est justement dû à l'échec de l'imagination. Pourtant, le récit chamsonien, à travers cette incompatibilité entre la réalité et la faculté langagière de décrire, fonctionne ici à l'instar de l'écart produit entre les signes verbaux et les éléments plastiques dans Ceci n'est pas une pipe de Magritte. Dans les deux cas, c'est « le fond de discours affirmatif»(Foucault, 1994: 650) qui est détourné : représenter l'image de la pipe alors même que l'énoncé verbal le nie, suggère, par cette négation même, une lecture autre qu'affirmative : ne pas décrire l'image réelle (cette fois non pas une pipe, mais un défilé nazi), mais la dessiner par la négation de mots/concepts ordinaires (ce sont des gens comme nous, mais il y a autre chose, ce sont bien des nationalistes, mais il y a encore autre chose etc.), dévoile en fin de compte ce que l'affirmation ou autrement dit une description (affirmative) n'aurait pas donné. C'est dans cet échec verbal du descriptif ordinaire (qui n'est pas ici l'indicible, tout au contraire) que se situe notamment « la vérité » sur la réalité dont le refus par Carrière constitue la "compréhension imaginative ${ }^{17}$, tout comme chez Magritte "Ceci est une pipe est bien devenu Ceci n'est pas une pipe » (Foucault, 1994 : 650).

\section{BIBLIOGRAPHIE}

ARENDT, Hannah (2002). Les origines du totalitarisme - Eichmann à Jérusalem. Paris : Gallimard.

BÉRAUD, Henri (1926). « Ce que j’ai vu à Berlin », Le Journal, 9 octobre, p. 1.

BÉRAUD, Henri (1941). « Et les juifs ? ", Gringoire, nº 633, 23 janvier.

BEUCLER, André (1933). « Berlin, la ville la plus anxieuse du monde », L'Intransigeant, n.. 19475, 21

février, p. 1.

BEUCLER, André (1933). « Le Reich d'Hitler », Le Petit Parisien, 3 octobre, p. 5.

CHAMSON, André (2005). Les livres de la guerre. Paris : Omnibus.

DECOUR, Jacques (2003). Philisterburg. Tours : farrago.

DORGELÈs, Roland (1937). « Les parias d'Hitler », L'Intransigeant, 26 janvier, pp. 1 et 5.

DORGELÈs, Roland (1937a). « La grande pénitence allemande », L'Intransigeant, 25 Janvier, p. 3.

DORGELÈs, Roland (2013). D'une guerre à l'autre. Paris : Omnibus. 
FAVRE, Pierre (2002). Jacques Decour. L'oublié des lettres françaises. Tours : farrago.

FOUCAULT, Michel (1973). Ceci n'est pas une pipe. « Lettre de Magritte à Michel Foucault », 4 juin 1966, Fontfroide-le-Haut : Fata Morgana. pp. 88-90.

FoucAult, Michel (1994). « Ceci n'est pas une pipe », Dits et écrits I. Paris : Gallimard. pp. 635-650.

FREDERIX, Pierre (1933). « Promenades et regards à travers le $\mathrm{III}^{\mathrm{e}}$ Reich. Racistes de Province », Le Petit Parisien, 25 décembre, pp. 1 et 5.

FREDERIX, Pierre (1934). « Promenades et regards à travers le $\mathrm{III}^{\mathrm{e}}$ Reich. La Paix de Hitler », Le Petit Parisien, 6 janvier, pp. 1 et 3.

JAVAKHISHVILI, MIKHEIL (2005). « LA CHAÎNE FONDUE », IN Euvres, VOL. 3, TBILISSI, « SAQARTVELOS MACNÉ ».

JOUANJAN, Olivier (2017). Justifier l'injustifiable. L'ordre du discours juridique nazi. Paris: PUF.

KIERAN, Matthew (1996). « Art, Imagination, and the Cultivation of Morals », The Journal of

Aesthetics and Art Criticism, vol. 54, pp. 337-351.

KLEMPERER, Victor (1996). LTI, La langue du III Reich. Paris : Albin Michel.

LARGUE, Jean (1935). « Le 'fait nazi'. Réflexions inspirées par le congrès de Nuremberg », La Revue

hebdomadaire, 2 novembre, p. 31.

MANN, Érika (2011). Quand les lumières s'éteignent. Paris : Grasset.

SALLÉE, Frédéric (2017). Sur les chemins de terre brune. Voyages dans l'Allemagne nazie, 1933-1939.

Paris : Fayard.

ZAMIATINE, Eugène (2010) [1920-1921], Nous autres. Édition électronique, “Ebooks libres et gratuits".

\section{NOTES}

1. Chez Evguéni Zamiatine, par exemple, c'est la présence de la fantaisie chez l'humain qui représente la menace primordiale. Considérée comme maladie par l'état total, la fantaisie ou autrement dit l'imagination doit être éradiquée par le biais de l'opération afin d'assujettir l'homme.

2. Il y a plusieurs niveaux d'étrangeté que Mann introduit ici : (1) l'étranger dans un lieu et dans une (2) atmosphère doublement étrangère car il ne connaît pas de régime semblable (donc, il doit imaginer au sens de généraliser ce qu'il a entendu avant sur l'Allemagne et ce qu'il vient de voir de ses propres yeux).

3. Voir le dialogue avec la jeune pharmacienne lors du bombardement de la ville (Klemperer, 1996 : 236-238).

4. L'absence de traitement de la question antisémite souvent observée, ou l'inadéquation de l'appréhension de la gravité du climat des persécutions par le voyageur étranger pourrait dépendre sur plusieurs facteurs. Pour Sallée, l'une des causes réside dans l'indifférence globale de la population allemande elle-même, observée par le voyageur, quant au traitement infligé aux Juifs ; mais elle peut aussi résider dans l'accueil réservé dans son propre pays à son récit, lorsque la question paraît excessivement soulignée : c'est qu'il semble alors appuyer d'une manière trop ciblée sur l'antisémitisme et omettre d'autres persécutions (Sallée $2017: 330$ et 339).

5. Ailleurs, Sallée note : «Quantité de voyageurs ont admis leur impuissance face à leur capacité à analyser correctement et de manière lucide le nazisme. Le bouleversement des repères politiques classiques opéré par Hitler a rendu la tâche ardue » (Sallée, 2017 : 59). Jean Largue, collaborateur 
auprès de La Revue hebdomadaire, observe lors du congrès de Nuremberg qu'il ne s'agit pas d'un sujet relevant du politique, mais d'un cas de figure transcendant le politique qui touche l'affect : «Avant d'être un fait politique c'est un fait affectif et ce fait consiste dans un lien sentimental de nature toute particulière entre le Führer et son peuple " (Largue, 1935 : 31, cité également in Sallée, $2017: 59$ ).

6. Ayant pris le parti des manifestants le 6 février 1934, il publie dans Gringoire, sous l'Occupation et lorsque se déroulent les persécutions, qu' « il faut être antisémite ", surtout parce que « le juif est l'ennemi-né des traditions nationales » (Béraud, 1941).

7. Les frères Tharaud, antisémites convaincus, remarquent, à leur tour la nouveauté du phénomène auquel ils se heurtent lors de leur voyage en Allemagne, en 1933: "Ce qui est menacé, à cette heure, ce ne sont ni les livres, ni la religion hébraïque ; ce n'est même pas le commerce. (...) Ce qui est menacé, c'est quelque chose qu'on ne peut pas brûler, qu'on ne peut ni acheter, ni vendre, et dont il est bien impossible à un Juif de se débarrasser : c'est sa race, son être même. (Cité in Sallée, 2017 : 348). Eux de même, entament leur jugement par la négation : la menace antisémite ne repose ni sur les livres, ni sur la religion, ni sur le métier, mais sur quelque chose d'absolument autre.

8. Même s'il s'agit, avec les frères Tharaud ou Beraud, d'écrivains antisémites ou convertis à l'antisémitisme, ces exemples nous paraissent intéressantes dans la mesure où elles répondent à une logique identique dans l'imagination du phénomène existant, le nazisme.

9. Cette réaction renvoie aux autres épisodes semblables dans le monde de fiction où l'auteur, afin de rendre la résistance à l'événement totalitaire, adopte la forme de rejet total, où l'inintelligibilité s'avère même à l'amont de ce rejet. Comme par exemple, la protagoniste de Javakhishvili qui s'écrit: «je ne comprends pas et ne veux pas comprendre!» (Javakhishvili, 2005 : 42). Comprendre l'irrationnel du fonctionnement de la machine soviétique ou nazie, équivaut à un certain degré d'acceptation de ce fonctionnement.

10. L'inaptitude de se faire comprendre, les mots lui manquant, c'est par le geste qu'il entreprend l'explication, et ce geste, c'est de frapper le poing sur la table.

11. Plus tard il apprend que le père était obligé de dire ce qu'il ne pensait pas, car les membres de famille se méfient les uns des autres, et le père contrôle chaque mot qu'il prononce devant un étranger afin que son fils ne le dénonce.

12. Souligné dans le texte.

13. Voici le descriptif du défilé qui précède le passage cité plus haut: «Ce matin, au réveil, j’ai entendu une sorte de cantique scandé par un bon chœur d'hommes. De ma fenêtre, j'ai vu un régiment défiler dans la grande rue, que l'on aperçoit par une rue transversale. Un régiment civil : blouses kaki, têtes nues, culottes courtes, grosses bottes. Ces êtres défilaient avec beaucoup d'ordre et de sérieux, en chantant très juste leur hymne mi-religieux, mi-guerrier. Le choc unique et multiple des bottes sur le pavé, l'ensemble dru de ces voix vigoureuses, l'aspect serein de ces muscles et des visages au repos donnaient une impression de puissance contenue » (Decour, 2003 : 68-69).

14. Voir Joseph Rovan, lorsqu'il est confronté lui aussi en 1932-1933 à cette fascination (Decour 2002 : 66).

15. Dans Philisterburg, il revient sur le thème : «La littérature ne les intéresse pas : à quoi sertelle donc? Ils lisent Bronner parce qu'ils y trouvent de la politique de parti, mais ils ne lisent pas Goethe pour diverses raisons : c'est froid et classique (ils veulent du piment) ; ce n'est pas actuel (les journaux sont leur pâture idéale) ; surtout, - ce qu'ils ne disent pas, - ils ne sont pas en état de comprendre les leçons de Goethe » (Decour 2003 : 124).

16. C'est nous qui soulignons.

17. Même si Carrière n'arrive pas à mettre les mots sur l'image (défilé etc.), il refuse tout court ce qu'il a pu voir pendant son bref séjour en Allemagne. 


\section{RÉSUMÉS}

Nous envisageons d'interroger les écrivains français et le regard qu'ils ont porté sur l'Allemagne nazie entre 1933 et 1939. Notre questionnement est le suivant : étant devenus témoins directs, ont-ils appréhendé le danger et la menace de l'état dictatorial, des lois raciales, des persécutions des Juifs ? Sous quelle forme littéraire ont-ils dénoncé les périls? Quelle est l'image, réelle ou imaginée, que ces écrivains conçoivent de l'Allemagne à l'emprise des défilés et des saluts nazis? Dans quelle mesure était-il possible d'imaginer ou d'appréhender la réalité dystopique qui se présentait à eux?

We intend to question the French writers on their perception of the Nazi Germany through the experience of travel in the country between 1933 and 1939. Our questioning is the following: having become direct witnesses, did they apprehend the danger and the threat of the dictatorial state as well as racial laws and persecutions of the Jews? In what literary form have they denounced the perils? What is the image, real or imagined, that these writers conceive of Germany in the grip of Nazi parades and salutes? To what extent was it possible to imagine or apprehend the dystopian reality that presented to them?

INDEX

Keywords : imagination, Nazism, French writers, travel, reality

Mots-clés : imagination, nazisme, écrivains français, voyage, réalité

\section{AUTEUR}

\section{ATINATI MAMATSASHVILI}

Université d'État Ilia (Tbilissi, Géorgie) / Aix-Marseille Université atinati_mamatsashvili[at]iliauni.edu.ge 